\title{
Can Low Intensity Resistance Training with Blood Flow Restriction be used for the Management of Lateral Elbow Tendinopathy?
}

ISSN: 2577-1914

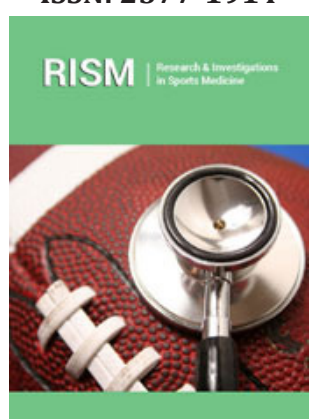

*Corresponding author: Stasinopoulos Dimitrios, Department of Physiotherapy, Faculty of Health and Caring Sciences, University of West Attica, Agiou Spyridonos 28, Egaleo 12243, Athens, Greece

Submission: 侮 July 06, 2020

Published: July 29, 2020

Volume 6 - Issue 5

How to cite this article: Stasinopoulos Dimitrios. Can Low Intensity Resistance Training with Blood Flow Restriction be used for the Management of Lateral Elbow Tendinopathy?. Res Inves Sports Med, 6(5): RISM.000647. 2020. DOI: $10.31031 /$ RISM.2020.06.000647

Copyright@ Stasinopoulos Dimitrios, This article is distributed under the terms of the Creative Commons Attribution 4.0 International License, which permits unrestricted use and redistribution provided that the original author and source are credited.
Stasinopoulos Dimitrios*

Department of Physiotherapy, University of West Atticam, Greece

\section{Editorial}

Lateral elbow tendinopathy (LET) commonly referred to as tennis elbow or lateral epicondylitis is one of the two most common tendinopathies of the upper extremity among athletes and non-athletes. Although the diagnosis of LET is simple, the ideal management for the rehabilitation of LET does not exist. A conservative approach is advocated by many physicians. Therefore, physical therapy is usually recommended. A plethora of physiotherapy modalities has been recommended for the management of LET such as heavy-slow resistance exercise, manual techniques, physical agents, external support and soft tissue manipulation. Such a plethora of techniques suggests that the ideal management strategy is unknown, and further research is required to find the most effective treatment strategy in LET patients.

The question that arises is why the optimal treatment of LET is still unknown, symptoms may persist for many months and recurrence is common. One possible answer is that should be used new techniques to improve patients' symptoms (pain, strength, and function). Lately, the use of low-intensity resistance training in combination with blood flow restriction (BFR) has been advocated as a clinically relevant rehabilitation tool for patients with tendinopathies such as LET. I wondered if low-intensity resistance training in combination with BFR could be an effective treatment approach in the rehabilitation of LET, thus the literature was searched.

An electronic search for clinical studies was carried out in six databases: Medline (from 1966 to March 2020), Embase (from 1988 to March 2020), Cinahl (from 1982 to March 2020), Index to Chiropractic literature (from 1992 to March 2020) SportDiscus (from 1990 to March 2020) and Chirolars (from 1994 to March 2020). A search took a very broad approach in order to capture all published material from any source including any clinical study, review, and letters to editors of journals. The following key words were used individually or in various combinations: "lateral elbow tendinopathy," "tennis elbow," "lateral epicondylitis," "lateral epicondylalgia," “extensor tendinopathy," “extensor tendonitis," “extensor tendinosis," "rehabilitation," "treatment," "management," "protocol," “optimal protocol," "blood flow restriction," " vascular occlusion," "strength training," "resistance training," “exercise training" and "kaatsu". Only English language publications were considered. Other references were attempted to identify from existing reviews, books and other papers cited in the publications searched. Additional reports were sought from the reference sections of papers that were retrieved, from contacting experts in the field, from the Cochrane Collaboration clinical trial register (last search March 2020) and from internet sites related to BFR. Unpublished reports and abstracts were included in the review. Just one published case study was found with positive effects [1]. However, due to its study design limits the generalization of these results. Future well-designed trials are needed to confirm the results of this case report establishing the effectiveness of such a treatment approach in the management of LET. 
One important factor, maybe the most, in order to determine the effectiveness of low-intensity resistance training in combination with BFR in the management of LET is to define the protocol in detail. The following parameters must be considered [2]:

1. Warming period

2. Frequency

3. Treatment period

4. Load

5. Restriction time

6. Sets

7. Cuff (type and width)

8. Repetition pressure (standardized or individualized?)

9. Rest between sets

10. Rest between exercises

11. Using a non pressure regulating cuff vs a pressure regulating cuff system

12. Patient position during LOP estimation

13. Restriction form (continuous or intermittent)

14. Execution speed (Tempo) with or without isometric pause?

15. Pain or not during execution

16. External paced audio or visual cue

17. Acute or chronic condition

18. Age of patient

19. Sex

It is worth to mention the heavy-slow resistance exercise programme for LET patients. Rehabilitation of LET is changing, and now eccentric training is not the only exercise option. Malliaras and his colleagues [3] concluded that clinicians should consider eccentric-concentric loading alongside or instead of eccentric loading. Martinez-Silvestrini et al. [4] stated that, unlike Achilles tendinopathy, LET is often related to forceful grip activities requiring isometric contraction, which would be more beneficial than the eccentric contraction in LET. Recently, isometric exercises have been recommended to reduce and manage tendon pain, increasing the strength at the angle of contraction without producing inflammatory signs [5]. The exercise program in LET should include exercises not only for extensor carpi radialis brevis (ECRB) strengthening, the most commonly affected structure, but also for supinator, rotator cuff, and scapular muscle strengthening [6,7]. Moreover, patients with LET have also reduced proprioception [8]. Techniques to improve reduced proprioception are also recommended. Finally, tendon neuroplastic training (TNT) is needed to combine isometric or isotonic strength training with an externally paced audio or visual cue [9]. The heavy-slow resistance exercise programme is individualized on the basis of the patient's description of pain experienced during the procedure [10]. The above can help researchers to define the optimal low-intensity resistance training in combination with the BFR protocol for the management of LET.

Finally, I wonder if low-intensity resistance training in combination with BFR can be delivered as a treatment in isolation in the management of LET. Heavy-slow resistance exercise programme is the most promising treatment approach in the management of LET. When the heavy-slow resistance exercise programme is applied as part of the rehabilitation process, combined with a range of physical therapy modalities such as electrotherapeutic modalities, manual therapy techniques, bracing/ taping and acupuncture, its effectiveness is higher than it is applied as monotherapy [1114]. Something similar might be true for low-intensity resistance training in combination with BFR. Further research is needed to consider this.

\section{References}

1. Canfield C (2018) Benefits of blood flow restriction training as an intervention for adults with lateral epicondylitis. Doctor of Physical Therapy.

2. Patterson SD, Hughes L, Warmington S, Burr J, Scott BR, et al. (2019) Blood flow restriction exercise: considerations of methodology, application, and safety. Front Physiol 10: 533.

3. Malliaras P, Barton C, Reeves N, Henning Langberg (2013) Achilles and patellar tendinopathy loading programmes: A systematic review comparing clinical outcomes and identifying potential mechanisms for effectiveness. Sports Med 43(4): 267-286.

4. Martinez-Silvestrini JA, Newcomer KL, Ralph E Gay, Michael P Schaefer, Patrick Kortebein, et al. (2005) Chronic lateral epicondylitis: comparative effectiveness of a home exercise program including stretching alone versus stretching supplemented with eccentric or concentric strengthening. J Hand Ther 18(4): 411-419.

5. Malliaras P, Cook J, Purdam C, Ebonie Rio (2015) Patellar tendinopathy: clinical diagnosis, load management, and advice for challenging case presentations. J Orthop Sports Phys Ther 45(11): 887-898.

6. Stasinopoulos D (2017) Strengthening of supinator in the management of lateral elbow tendinopathy. AMJ 10: 373-374.

7. Stasinopoulos D (2017) Scapular and rotator cuff strengthening in patients with lateral elbow tendinopathy. Hong Kong Physiother J 37: $25-26$.

8. Juul-Kristensen B, Lund H, Hansen K, Christensen H, DanneskioldSamsøe B, et al. (2007) Poorer elbow proprioception in patients with lateral epicondylitis than in healthy controls: a cross-sectional study. J Shoulder Elbow Surg 17(1 Suppl): 72S-81S.

9. Rio E, Kidgell D, Moseley GL, Gaida J, Docking S, et al. (2015) Tendon neuroplastic training: changing the way we think about tendon rehabilitation: a narrative review. Br J Sports Med 50(4): 209-215.

10. Stasinopoulos D, Stasinopoulos I (2017) Comparison of effects of eccentric training, eccentric-concentric training, and eccentricconcentric training combined with isometric contraction in the treatment of lateral elbow tendinopathy. J Hand Ther 30(1): 13-19.

11. Bisset LM, Vicenzino B (2015) Physiotherapy management of lateral epicondylalgia. J Physiother 61(4): 174-181.

12. Sims SE, Miller K, Elfar JC, Hammert WC (2014) Non-surgical treatment of lateral epicondylitis: a systematic review of randomized controlled trials. Hand (N Y) 9(4): 419-446. 
13. Weber C, Thai V, Neuheuser K, Groover K, Christ O (2015) Efficacy of physical therapy for the treatment of lateral epicondylitis: a metaanalysis. BMC Musculoskelet Disord 16: 223.
14. Stasinopoulos D, Stasinopoulou K, Johnson MI (2005) An exercise programme for the management of lateral elbow tendinopathy. $\mathrm{Br} \mathrm{J}$ Sports Med 39(12): 944-947.

\section{For possible submissions Click below:}

\section{Submit Article}

